Mit Salpetersäure erhitzt, liefert Carnin, an der Luft opak werdend, Krystalle von salpetersaurem Sarkin, dessen Formel Weidel $=\mathrm{C}^{5} \mathrm{H}^{4} \mathrm{~N}^{4} \mathrm{O}, \mathrm{NHO}^{3}$ fand. In den Mut-

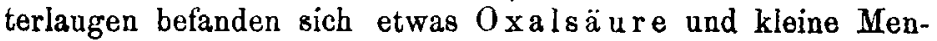
gen eines undeutl. kryst. gelben Körpers. Empirisch genommen, unterscheidet sich Carnin von Sarkin durch den Betrag von $\mathrm{C}^{2} \mathrm{H}^{4} \mathrm{O}^{2}$ (Essigsäure), denn $\mathrm{C}^{7} \mathrm{H}^{8} \mathrm{~N}^{4} \mathrm{O}^{3}-\mathrm{C}^{5} \mathrm{H}^{4} \mathrm{~N}^{4} \mathrm{O}$ $=\mathrm{C}^{2} \mathrm{H}^{4} \mathrm{O}^{2}$, Carnin minus Sarkin gleich Essigsäure. Aber weder Essigsäure noch Monobromessigsäure war nachweisbar.

Erwärmt man kleine Mengen von Sarkin mit frischem Chlorwasser und einer Spur Salpetersëure, so lange, bis die schwache Gasentwickelung, die sich einstellt, aufgehört hat, verdampft dann im Wasserbade vorsichtig zur Trockne und setzt den weissen Rückstand unter einer Glocke einer $\mathrm{H}^{3} \mathrm{~N}$ Atmosphäre aus, so färbt sich derselbe in kurzer Zeit dunkelroth. Dieselbe Reaction giebt das Carnin; sie ist hier auf Rechnung des Sarkins zu setzen, welches sich bei der Behandlung mit Chlor und $\mathrm{NO}^{5}$ erst bildet.

Im Fleischextracte fand Dr. H. Weidel auch Bernsteinsäure, die bis jetzt darin noch nicht aufgefunden war. Die auf die physiologische Wirkung des Carnins bezügl. Versuche von Brücke sind noch nicht zahlreich genug, um aus ihnen die letzten Schlüsse zu ziehen. Hlasi wetz theilt dieselben an a. 0 . mit. $H . L$.

\title{
Ueber die Proteinstoffe
}

haben H. Hlasiwetz und J. Habermann Untersuchungen veröffentlicht, welche die nahen Beziehungen dieser Substanzen zu den Kohlehydraten darthun. Schon Hunt hat (1847) ausgesprochen, dass man das Fibrin als das Nitril der Cellulose, Albumin und Caseïn als Nitrile des Dextrins und Gummi's und den Leim als das Nitril des Krümelzuckers betrachten könne. Später (1860) hat Schoonbrodt Versuche angekündigt, die es möglich erscheinen lassen sollten, Zucker zu eiweissartiger Substanz umzuwandeln.

Es hat indess weder Hunt seine Ansicht experimentell begründet, noch ist Schoonbrodt's versprochene Arbeit seither erschienen. Ohne thatsächliche Beweise können aber Ansichten dieser Art nicht zu Ueberzeugungen werden. Was in dieser Richtung etwa verwerthbar wäre, beschränkt sich 
auf einige Versuche Śchützenberger's über die Einwirkung des $\mathrm{H}^{3} \mathrm{~N}$ auf Kohlehydrate bei höherem Druck in der Hitze, wodurch er amorphe Substanzen erhielt, die einen Stickstoffgehalt ron 2 bis 4 pr. C. zeigten und beim Erhitzen den Geruch nach verbranntem Horn verbreiteten.

Nach Hlasiwetz und $H$ abermann gewähren die bei gewissen Einwirkungen constant auftreterden Zersetzungsproducte der Proteinstoffe ziemlich bestimmte Andeutungen über die Natur der an der Bildung derselben betheiligten Verbindungen, Andeutungen, die es wahrscheinlich machen, dass sie auf die vorausgehende oder mindestens gleichzeitige Entstehung einiger anderer Verbindungen angewiesen sind; diese anderen sind offenbar nur die Kohlehydrate.

Beide Körpergruppen, Kohlehydrate und Proteïnstoffe umschliessen eine Anzahl unter einander isomerer und polymerer Substanzen. Einige derselben sind löslich und nicht organisirt, andere derselben sind unlöslich und organisirt.

Den löslichen (Dextrin, Eiweiss u. s. w.) wohnt eine "virtuclle Plasticitït" oder "Organisationsfähigkeit" inue, die in dem Spiele vitaler Processe zur Erscheinung kommt.

Die organisirten unlöslichen (Cellulose, thierisches Zellgewebe, Hornsubstanz u.s. w.) entstehen, wie man aunehmen muss, aus den löslichen nicht organisirten; dabei scheint sich das Molekul zu verdichten.

Die intermediäre Modification dieser Zustände scheint das Protoplasma, die werdende rudimentäre Zelle zu sein. Alles weist daranf hin, dass ein bestimmtes Abhängigkeitsverhältniss dieser beiden Körpergrnppen besteht, dass die Proteïnstoffe nicht verstanden werden können, wenn man nicht die Verhältnisse der Kohlehydrate mit in Rechnung bringt. Unter der Annahme aber, die Kohlehydrate seien das Primäre und die Proteïnstoffe Derivate derselben, bieten sich eine Reihe unverkennbarer Parallelen.

Schon die äussere Beschaffenheit beider Arten von Verbindungen bietet Analogieen. Sie ist am Aehnlichsten zwischen den Schleime bildenden Kohlehydraten und den thierischen Schleimen. ${ }^{*}$ )

*) Man vergleiche in der von mir bearbeiteten 2. Auflage von Marquart's Lebrbuch der Pharmacic. III. Bd. Organ. Chem. Präparate, Siebente Gruppe: Schleimstoffe oder organische Colloidsub. gtanzen.

H. Luduig. 
Eingetrocknetes Eiweiss, löslich gemachtes und dann getrocknetes Fibrin und Caseïn gleichen dem Gummi und Dextrin. Kohlehydrate organisiren sich zu einzelnen unzusammenhängenden Gebilden in den Amylonarten, die Proteïnkörper in den verschiedenen Arten von Blutzellen (und Dotterkörperchen. Eudwig).

Dem Protoplasma der Pflanzen entspricht die Granulose der Thiere, der pflanzlichen Cellulose das thierische Zellgewebe, der in den Schalen und Kernen der Pflanzen verdichte. ten Cellulose entspricht die Hornsubstanz, den krystallisirten Proteinkörpern der Pflanzen (z. B. in Lathraea Squamaria, nach Radlk ofer, in den Kartoffeln, nach $\mathrm{Cohn}$ ) das Hämatokrystallin der Thiere.

Die Erscheinung des Quellen's ohne Lösung mancher Proteinstoffe (bei Caseïn, Fibrin u. a.) scheint im Zusammenhange zu stehen mit derselben Erscheinung bei einigen Varietäten der Gummiarten (Bassorin, Traganth u. a.).

Die Löslichkeit mancher Gummiarten ist bedingt durch kleine Mengenalkalisch or Basen; jene worden aus solcher Lösung gefällt durch Zusatz von Säuren. Auch das Eiweiss verdankt, wenn es löslich ist, diese Löslichkeit vornehmlich kleinen Mengen alkalischer Verbindungen. Lösliches Serumeiweiss lässt sich frei von Alkalien oder Salzen gar nicht erhalten. Nimmt man durch Säuren oder andere Reagentien diese hinweg, so coagulirt das Eiweiss, wie etwa in gleichem Falle eine Arabinlösung.

Unlösliche Kohlehydrate, Amylon z. B. gehen, ohne ihre procent. Zusammensetzung zu ändern, durch anhaltendes Kochen mit Wasser, $\mathrm{ZnCl}$, Eisessig u. drgl. in lösliche Modificationen über; in derselben Weise können unlösliche Proteïnstoffe, wie Fibrin, löslich gemacht werden.

Auch der umgekehrte Fall ist bekannt: man kann das sonst lösliche Dextrin in einer unlöslichen Modification erhalten (vergl. Musculus, Zeitschr. für Chem. $1869,446$ und 1870,346$)$ und es dann dem unlöslichen Fibrin vergleichen, welches aus löslichem Albumin hervorgegangen ist.

Die Fähigkeit, durch Gährung zersetzt zu werden, zeichnet vor allen übrigen organ. Verbindungen gewisse Kohlehydrate und die Proteïnstoffe aus.

Die hauptsächlichsten Gährung s product e beider Körpergruppen stehen in einer unverkennbar sehr einfachen 
Beziehung zu einander. Man hat unter ihuen vornehmlich gefunden:

Aus Kohlehydraten: : Aus Proteinstoffen:

Kohlensäure, Wasserstoff, Aethylalkohol, Propylalkohol, Butylalkohol, Amylalkohol,

Glycerin, Essigsäure, Propionsäure, Buttersäure, Valeriansäure, Milchsäure, Bernsteinsäure. Kohlensäure, Wasserstoff, $\mathrm{HS}, \mathrm{H}^{3} \mathrm{~N}$, Aethylammin, Trimethylammin, Amylammin, Caproylammin *), (?) Essigsäure, Propionsäure (:), Buttersüure, Valeriansäure, Milchsäure, Leucin.

'Tyrosin.

Hier finden wir also entweder identische Producte, Glieder der Fettsäuren-Reihe, oder es sind bei den Kohlehydraten Alkohole, bei den Proteïnstoffen die den Alkoholen entsprechenden Ammine.

Vielleicht wird man bei näherer Untersuchung auch die der Essigsäure entsprechende Oxysäure (die Gly kolsäure) auffinden, da die Oxysäure der Propionsäure (die Milch$\mathrm{s} \ddot{\mathrm{ur}} \mathrm{e}$ ) in beiden Fällen vorkommt. (Im Traubensafte wurde durch Erlenmeyer das Vorkommen der Glykolsäure schon constatirt). Nur fiur das T y ro s in der Proteinstoffe, eine der aromatischen Reihe angehörige Verbindung lässt sich keine correspondirende stickstofffreie Verbindung unter den Gährungsproducten der Kohlehydrate finden.

Auch bei den übrigen Zersetzungsweisen der Proteïnstoffe treten immer gewisse Producte auf, die der aromatischen Reihe und andere, die auch den Kohlehydraten eigen sind.

*) Alex. Müller und O. Hesae finden diese Base in gefaulter Hefe, Sulliva in in gefaultem Mehl. (Juliresb. f. Chem. 1857, 402 und fiir 1858,230 .) 
Die Behandlung mit Salpetersäure hat geliefert: aus Kohlehydraten: aus Proteinstoffen:

\begin{tabular}{|c|c|c|}
\hline $\begin{array}{l}\text { Oxalsäure, } \\
\text { Aepfelsäure, } \\
\text { Weinsäure, } \\
\text { Zuckersäure, } \\
\text { Schleimsäure. }\end{array}$ & $\mid \begin{array}{l}\text { Oxalsäure, } \\
\text { Fumarsäure, } \\
\text { Zuckersäure. } \\
\text { (Berzelius). }\end{array}$ & $\begin{array}{r}\text { Nitr. Derivate }(\mathrm{X} \text { a n- } \\
\text { th oprote in s.); } \\
\text { dann mit NO }{ }^{5} \text { und } \\
\text { HClSubstitutions- } \\
\text { producte mit NO } \\
\text { u. Cl, wahrschein- } \\
\text { lich Derivate der } \\
\text { Oxy - und Para- } \\
\text { oxybenz oësäu- } \\
\text { re, die ein Spal- } \\
\text { tungsproduct des } \\
\text { Ty rosins ist. }\end{array}$ \\
\hline
\end{tabular}

Die Oxydation mit $\mathrm{MnO}^{2}$ und $\mathrm{SO}^{3}$ gab: aus Kohlehydraten: Ameisensäure, Essigsäure, aus Proteïnstoffen:

Ameisensäure, Essigsäure und deren Homologe. Benzo ësäure, bis hinauf zur Ca- (Fröhde's, aus prylsäure, Leim erhalt. Col-

Aldehyd,

Acroleïn.

Aldehyde dieser linsäure war unSäuren, Acetonitril, Propionitril, Valeronitril.

Durch Behandlung mit Schwefelsäure wurden erhalten: aus Kohlehydraten: Ameisensäure, Glycinsäure,

Gepaarte säuren, (Sulfosäuren). aus Proteinstoffen:

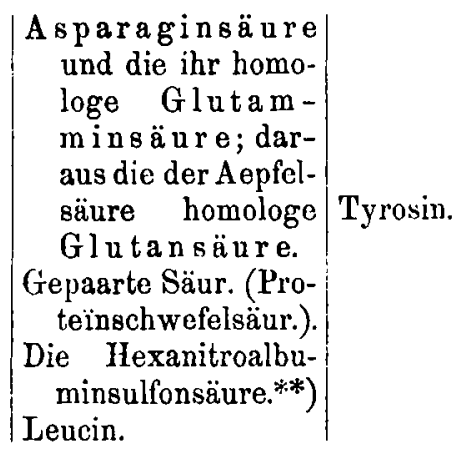
und die ihr homologe Glutammins äure; daraus die der A epfelsäure homologe Tyrosin. Glutansäure. Gepaarte Säur. (Proteïnschwefelsäur.). Die Hexanitroalbuminsulfonsäure.**) Leucin. reine Benzoës.); Benzaldehyd. 
Mit Kalihydrat gesclumolzen gaben: die Kohlehydrate: die Proteinstoffe:

Oxalsäure, Essigsäure, Propionsäure, Acetone (Ketone), Bernsteinsäure,

\section{Humussubstanzen.}

Oxalsäure,
Essigsüure,
Buttersäure,
Valeriansäure,
Leucin,
sauerstoffreie
Amminbasen,
Humussubstanzen.

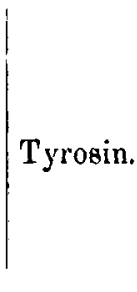

Mit Kalibydrat gekocht, liefern die Proteinsubstanzen auch Glykokoll.

Jod und doppeltkohlensaures Kali geben: mit Kohlehydraten: mit Proteinsubstanzen: Jodoform. Jodoform.

Die trockene Destillation liefert: aus Koblehydraten:

$\mathrm{CO}^{2}$, gasformige Kohlenwasserstoffe,

Methylalkohol,

Essigsäure,

Ketone,

Phenol,

Guajacol, Kresol,

Brenzcatechin,

Kohlenwasserstoff der a ro ma-

tis chen Reihe,

Naphthalin, Chrysen,

Paraffin.

Die vorstehende Zusammenstellung zeigt, wie scharf begrenzt die aus den Kohlehydraten und den Proteïnstoffen gewinnbaren Zersetzungsproducte sind, was sie Gemeinsames und was sie Verschiedenes haben und wie die eine Reihe der von den Proteïnstoffen abstammenden Producte immer, wo nicht identisch, so doch aufs Nächste verwandt mit der der Kohlehydrate ist.

Rechnet man dazu, dass thierische Stoffe, wie das Mucin und $\mathrm{Hy}$ alin, mit verdünnten Säuren gekocht neben Proteinstoffen Traubenzucker liefern, dass das Chitin und Cerebrin als Glykoside bctrachtet werden können; erwägt man, dass Proteïnstoffe in Pflanzen und Thieren fast 
inmer mit Kohleliydraten zusammen vorkommen; berïcksichtigt man endlich, dass, wie die Physiologie in der letzten Zeit aus den Ernährungs - und Fütterungs - Versuchen schliesst, die Proteinstoffe ebensowohl zur Fettbildung dienen, als sie zum Ersatz des abgenutzten Muskels und der Gewebe verwendet werden; so wird es mehr als wahrscheinlich, dass die Proteïnstoffe und Kohlehydrate in einer genetischen Beziehung zu einanderstehen.

Diese Anschauung wird nun auch durch die neuen Versuche von Hlasiwetz und Habermann unterstützt, bei denen sie eine Reihe von Proteïnstoffen (Hühnereiweiss, Caseïn, Fibrin, Pflanzeneiweiss, Legumin, Pflanzenfibrin und Gliadin aus Klebor) mit Brom und Wasser im verschlossenen Glasgefässen erhitzten und die entstandenen Producte analysirten. Sie konnten so die Proteïnsubstanzen total, ohne Rest, zersetzen.

In den beim Oeffnen der Flaschen entweichenden Gasen fand sich vornehmlich $\mathrm{Kohlens} \ddot{a} u r^{\mathrm{e}}$, unter den nicht flüchtigen Producten fand sich Phosphorsäure. Als organische Zersetzungsproducte der Proteinstoffe erhielten sie:

Bromessigsäure,| Asparaginsäure,| Leucin, |TribromamidoBromoform.

$\mid$\begin{tabular}{l|l|l} 
Malaminsäure, & Leucimid, \\
$?$ & Capronsäure, & benzoësäure, \\
Oxalsäure. & $?$ & Bromanil.
\end{tabular}

Auch war etwas Ammoniak und humöse Substanz gebildet worden. Niemals fanden sie Tyrosin. Da nach Städeler durch Chlor das Tyrosin völlig in Chloranil und Chloraceton rerwandelt wird, so jst kein Zweifel, dass das Brom in ähnlicher Weise wirke und dass das erhaltene Bromanil und die Tribromamidobenzoësäure aus derselben Quelle stammt, aus welcher sonst Tyrosin hervorgeht. (Nach Barth liefert Tyrosin, mit KO, HO geschmolzen, Paraoxybenzoësäure.)

Von 100 Theilen trockner Proteïnsubstanz wurden erhalten:

\begin{tabular}{|c|c|c|c|c|c|}
\hline Bromoform & $\begin{array}{l}\text { aus Eier- } \\
\text { albumin. } \\
29,9\end{array}$ & $\begin{array}{l}\text { Pflanzen } \\
\text { albumin. } \\
39,1\end{array}$ & $\begin{array}{c}\text { Caseïn. } \\
37,0\end{array}$ & $\begin{array}{c}\text { Legumin } \\
44,9\end{array}$ & heile. \\
\hline Bromessigsäure & 22,0 & 16,9 & 22,1 & 26,2 & $"$ \\
\hline Oxalsäure & 12,0 & 18,5 & 11,2 & 12,5 & , \\
\hline $\begin{array}{l}\text { Asparaginsäure u. } \\
\text { als Malaminsäure } \\
\text { angenomm. Säure }\end{array}$ & 23,8 & 23,1 & 9,3 & 14,5 & $"$ \\
\hline Leucin (rohes) & 22,6 & 17,3 & 19,1 & 17,9 & ” \\
\hline Bromanil & 1,5 & 1,4 & 0,3 & 1,4 & $"$ \\
\hline
\end{tabular}


Dass bei dieser Behandlung keine Glykonsäure orhalten wird (die man unter ähnl. Umständen aus verschiedenen Zuckerarten bekommt), hat darin seinen Grund, dass durch weitere Behandlung mit Brom, Wasser etc. die Glykonsäure fast gerade aut in $\mathrm{CO}^{2}, \mathrm{Bromoform}, \mathrm{Bromessig-}$ s äure und Oxals äure zerlegt wird.

Die Zersetzung der Proteïnstoffe durch Brom und Wasser hat demnach in Uebereinstimmung mit den anderen Zersetzungsweisen wieder zu Producten geführt, die sich in die fette und in die aromatische Gruppe einreihen lassen. Betrachtet man die gefundenen Glieder der Gruppe näher, so erkennt man, dass die höchsten nicht mehr als sechs A tome Kohlenst off $(\mathrm{C}=12)$ im Kerne oder in der Hauptkette enthalten, so dass man sie auf die beiden Kohlenwasserstoffe $\mathrm{C}^{6} \mathrm{H}^{14}$ und $\mathrm{C}^{6} \mathrm{H}^{6}$ zurückführen kann.

Characteristisch für die Proteinstoffe sind nur die höchsten Glieder: Leucin, Tyrosin, Asparaginsäure, und Glutaminsäure; alle übrigen sind nur Abkömmlinge dieser, sie sind secundäre Producte.

Uebersichtliche Zusammenstellung der Zersetzungsproducte:

A. Von $\mathrm{C}^{6} \mathrm{H}^{14}=\left(\mathrm{CH}^{3} \_\mathrm{CH}^{2} \_\mathrm{CH}^{2} \smile \mathrm{CH}^{2} \_\mathrm{CH}^{2} \smile \mathrm{CH}^{3}\right)$ lassen sich ableiten:

I. Caprylamin $\mathrm{C}^{6} \mathrm{H}^{13}\left(\mathrm{H}^{2} \mathrm{~N}\right)$; Amylamin $\mathrm{C}^{5} \mathrm{H}^{11}\left(\mathrm{H}^{2} \mathrm{~N}\right)$; Butylamin $\mathrm{C}^{4} \mathrm{H}^{9}\left(\mathrm{H}^{2} \mathrm{~N}\right)$; Propylamin $\mathrm{C}^{3} \mathrm{H}^{7}\left(\mathrm{H}^{2} \mathrm{~N}\right)$; Aethylamin $\mathrm{C}^{2} \mathrm{H}^{5}\left(\mathrm{H}^{2} \mathrm{~N}\right)$ and Methylamin $\mathrm{CH}^{3}\left(\mathrm{H}^{2} \mathrm{~N}\right)$.

II. Capronsäure $\mathrm{C}^{5} \mathrm{H}^{11}(\mathrm{COOH})$; Valerians. $\left(\mathrm{C}^{4} \mathrm{H}^{9}(\mathrm{COOH})\right.$; Buttersäure $\mathrm{C}^{3} \mathrm{H}^{7}(\mathrm{COOH})$; Propionsäure $\mathrm{C}^{2} \mathrm{H}^{5}(\mathrm{COOH})$; Essigsäure $\mathrm{CII}^{3}(\mathrm{COOH})$.

III. Leucin $\mathrm{C}^{5} \mathrm{H}^{10}\left\{\begin{array}{l}\mathrm{H}^{2} \mathrm{~N} \\ \mathrm{COOH}\end{array} ;\right.$ Glykokoll $\mathrm{CH}^{2}\left\{\begin{array}{l}\mathrm{H}^{2} \mathrm{~N} \\ \mathrm{COOH}\end{array}\right.$.

IV. Milchsäure $\mathrm{C}^{2} \mathrm{H}^{4}\left\{\begin{array}{l}\mathrm{OH} \\ \mathrm{COOH}\end{array}\right.$

V. Glutaminsäure $\mathrm{C}^{3} \mathrm{H}^{5} \mathrm{H}^{2} \mathrm{~N}\left\{\begin{array}{l}\mathrm{COOH} \\ \mathrm{COOH}\end{array}\right.$ Asparaginsäure $\mathrm{C}^{2} \mathrm{H}^{3} \mathrm{H}^{2} \mathrm{~N}\left\{\begin{array}{l}\mathrm{COOH} \\ \mathrm{COOH}\end{array}\right.$

VI. Fumarsäure $\mathrm{C}^{2} \mathrm{H}^{2}\left\{\begin{array}{l}\mathrm{COOH} \\ \mathrm{COOH}\end{array}\right.$

VII. Oxalsäure $\left\{\begin{array}{l}\mathrm{COOH} \\ \mathrm{COOH}\end{array}\right.$

VIII. Kohlensäure $=\mathrm{CO}^{2}$. 
B. Von $\mathrm{C}^{6} \mathrm{H}^{6}=\left(\beth^{*} \mathrm{CH}=\mathrm{CH} \_\mathrm{CH} O \mathrm{CH} \_\mathrm{CH} C \mathrm{CH} \Psi^{*}\right)$ lassen sich ableiten :

1. Tyrosin $\mathrm{C}^{6} \mathrm{H}^{3}\left\{\begin{array}{l}\mathrm{HN}, \mathrm{C}^{2} \mathrm{H}^{5} \\ \mathrm{OH} \\ \mathrm{COOH}\end{array}\right.$ ?

II. Benzoësäure $\mathrm{C}^{6} \mathrm{II}^{5}(\mathrm{COOH})$; Paraoxybenzoësäure $=$ $\mathrm{C}^{6} \mathrm{H}^{4}\left\{\begin{array}{l}\mathrm{COOH} \\ \mathrm{OH}\end{array}\right.$ (daraus Substitutionsproducte mit $\mathrm{Cl}$ und $\mathrm{NO}^{2}$ ). III. Benzaldehyd $\mathrm{C}^{6} \mathrm{H}^{5}(\mathrm{COH})$.

IV. Chinon $\mathrm{C}^{6} \mathrm{H}^{4} \mathrm{O}^{2}$ (daraus Bromanil). (Annalen d. Chem. u. Pharm. Sept. 1871, Bd. 159, S. 304333.).

H. L.

\section{Roggenmehl auf Gehalt an Mutterkorn zu prïfen.}

Nach Prof. Böttger überschüttet man eine Probe des fraglichen Mehles mit seinem gleichen Volumen Essigäther,

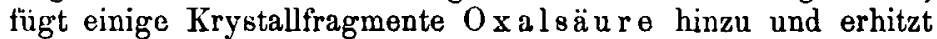
das Gemisch einige Minuten lang zum Kochen. Erscheint die Flüssigkeit nach dem Erkalten röthlich gefärbt, so ist Mutterkorn vorhanden. (Zeitschr. d. allgem. österrcich. Apoth.Vereins. 1. Sept. 1871. Nr. 25, S. 638.). $H, L$.

\section{Prüfung des Brodes auf Alaun.}

Sie geschieht nach Carter Moffat durch Befeuchtung desselben mit einer aus $\mathrm{Campecheholz}$ bereiteten Tinctur, welche das Vorhandensein von Ala un durch eine dunkelrothe Färbung verräth, während unverfälschtes Brod sich mit dieser Tinctur nur strohgelb färbt. (The Pharmacist and Chemic. Record, Chicago, Jul. 1871. P. 157.). Wp. 